\title{
SPLIT VARIATIONAL INCLUSIONS FOR BREGMAN MULTIVALUED MAXIMAL MONOTONE OPERATORS
}

\author{
Mujahid AbBas ${ }^{1}$, FAIK Gürsoy ${ }^{2, *}$, Yusuf Ibrahim ${ }^{3}$ And Abdul Rahim Khan ${ }^{4}$
}

\begin{abstract}
We introduce a new algorithm to approximate a solution of split variational inclusion problems of multivalued maximal monotone operators in uniformly convex and uniformly smooth Banach spaces under the Bregman distance. A strong convergence theorem for the above problem is established and several important known results are deduced as corollaries to it. As application, we solve a split minimization problem and provide a numerical example to support better findings of our result.
\end{abstract}

Mathematics Subject Classification. 47J25.

Received January 27, 2020. Accepted July 29, 2020.

\section{INTRODUCTION}

Censor [8] imposed the well known split feasibility problem (SFP), which is formulated as finding a point $x^{*} \in C$ such that $A x^{*} \in Q$, where $C$ and $Q$ are nonempty closed and convex subsets of $\mathbb{R}^{n}$ and $\mathbb{R}^{m}$, respectively, where $A$ is an $m \times n$ matrix. Byrne [3], defined CQ-algorithm as follows:

$$
x_{n+1}=P_{C}\left(x_{n}+\gamma A^{T}\left(P_{Q}-I\right) A x_{n}\right), n \geq 0,
$$

where $x_{0} \in \mathbb{R}^{n}$ is an initial value, $\gamma \in\left(0, \frac{2}{\|A\|^{2}}\right)$ and $P_{C}$ and $P_{Q}$ denote the metric projections onto $C$ and $Q$, respectively. The split feasibility problem has been considered by many authors and in many aspects $[1-3,5,8,9,13,16,25,26,30]$. In practice, SFP serves as a model in the intensity-modulation radiation therapy (IMRT) treatment planning [2,5]. Censor et al. [10] introduced a concept of Split Variational Inequality Problem (SVIP), which is a problem of finding a point $x^{*} \in H_{1}$ solves

$$
\left\langle f\left(x^{*}\right), x-x^{*}\right\rangle \geq 0 \text { for all } x \in C,
$$

and the point $y^{*}=A x^{*} \in H_{2}$ such that

$$
\left\langle g\left(y^{*}\right), y-y^{*}\right\rangle \geq 0 \text { for all } y \in Q
$$

Keywords. Split variational inclusion problem, maximal monotone operators, Bregman distance, strong convergence, uniformly convex and uniformly smooth Banach space.

1 Department of Mathematics, Government College University, Katchery Road, Lahore 54000, Pakistan.

2 Department of Mathematics, Adiyaman University, Adiyaman 02040, Turkey.

3 Department of Mathematics, Sa'adatu Rimi College of Education, Kumbotso Kano, P.M.B. 3218 Kano, Nigeria.

4 Department of Mathematics and Statistics, King Fahd University of Petroleum and Minerals, Dhahran 31261, Saudi Arabia.

* Corresponding author: faikgursoy02@hotmail.com 
where $C$ and $Q$ are closed and convex subsets of Hilbert spaces $H_{1}$ and $H_{2}$, respectively, $A: H_{1} \rightarrow H_{2}$ is a bounded linear operator and $A^{*}: H_{2} \rightarrow H_{1}$ is adjoint of $A, f: H_{1} \rightarrow H_{1}$ and $g: H_{2} \rightarrow H_{2}$ are two given operators. Furthermore, they proposed the following algorithm. Let $\lambda>0$ and $x_{1} \in H_{1}$ be arbitrary chosen. Define the sequence $\left\{x_{n}\right\}$ by

$$
\left.x_{n+1}=P_{C}^{f, \lambda}\left(x_{n}+\gamma A^{*}\left(P_{Q}^{g, \lambda}-I\right) A x_{n}\right)\right), \forall n \geq 0,
$$

where $\gamma \in\left(0, \frac{1}{\|A\|^{2}}\right)$, and denoted by $P_{C}^{f, \lambda}$ and $P_{Q}^{g, \lambda}$ the expressions $P_{C}(I-\lambda f)$ and $P_{Q}(I-\lambda g)$, respectively. By some assumptions imposed on the operators $f$ and $g$, they proved weak convergence result for the sequence $\left\{x_{n}\right\}$ to a solution point of split variational inequality problem.

Let $E$ be a real normed space with dual $E^{*}$ and $J(x)=\left\{x^{*} \in E^{*} ;\left\langle x, x^{*}\right\rangle=\|x\|\left\|x^{*}\right\|,\left\|x^{*}\right\|=\|x\|\right\}$ be the normalized duality. A map $B: E \rightarrow E^{*}$ is called monotone if for each $x, y \in E$, the following inequality holds: $\langle\eta-\nu, x-y\rangle \geq 0 \forall \eta \in B x, \nu \in B y$. It is called maximal monotone if, in addition, the graph of $B$ is not properly contained in the graph of any other monotone operator. Also, $B$ is maximal monotone if and only if it is monotone and for all $t>0, R(J+t B)=E^{*}$, where $R(J+t B)$ is the range of $(J+t B)$; see [4]. By using maximal monotone mappings, Moudafi [15] introduced the following Split Monotone Variational Inclusion (SMVI).

$$
\left\{\begin{array}{l}
\text { find } x^{*} \in H_{1}: 0 \in f\left(x^{*}\right)+B_{1}\left(x^{*}\right), \text { and } \\
y^{*}=A x^{*} \in H_{2}: 0 \in g\left(y^{*}\right)+B_{2}\left(y^{*}\right),
\end{array}\right.
$$

where $B_{1}: H_{1} \rightarrow 2^{H_{1}}$ and $B_{2}: H_{2} \rightarrow 2^{H_{2}}$ are multi-valued maximal monotone mappings on Hilbert spaces, $H_{1}$ and $H_{2}$, respectively, and $A: H_{1} \rightarrow H_{2}$ is a bounded linear operator, $f: H_{1} \rightarrow H_{1}$ and $g: H_{2} \rightarrow H_{2}$ are two given single-valued operators. When $f$ and $g$ are zero functions in (1.2), we have the usual Split Variational Inclusion Problem (SVIP). The algorithm introduced by Schöpfer et al. [20] involves computations in terms of Bregman distance in the setting of p-uniformly convex and uniformly smooth real Banach spaces. Their iterative algorithm given below, converges weakly under some suitable conditions.

$$
x_{n+1}=\Pi_{C} J^{-1}\left(J x_{n}+\gamma A^{*} J\left(P_{Q}-I\right) A x_{n}\right), n \geq 0,
$$

where $\Pi_{C}$ denotes the Bregman Projection and $A^{*}$ the adjoint operator of $A$. It is obvious that, strong convergence is more useful than the weak convergence in some applications. Recently, strong convergence theorems for SFP have been studied in the setting of p-uniformly convex and uniformly smooth real Banach spaces; see for example $[11,17,22,23]$.

In this paper, inspired by the above cited works, we use a modified version of (1.1) and (1.3) to approximate a solution of the problem proposed here. Both the iterative methods and the underlying space used here are improvements of those employed in $[6,7,10,11,13,17,20,22,23,28]$ and the references therein.

Definition 1.1. For each $p>1$, let $g: \mathbb{R}^{+} \longrightarrow \mathbb{R}^{+}$given by $g(t)=t^{p-1}$ be a gauge function such that $g(0)=0$ and $\lim _{t \rightarrow \infty} g(t)=\infty$. We define the generalized duality map $J^{p}: E \longrightarrow 2^{E^{*}}$ given by

$$
J_{g(t)}=J^{p}(x)=\left\{x^{*} \in E^{*} ;\left\langle x, x^{*}\right\rangle=\|x\|\left\|x^{*}\right\|,\left\|x^{*}\right\|=g(\|x\|)=\|x\|^{p-1}\right\} .
$$

Definition 1.2. Let $E$ be a smooth Banach space, the Bregman distance $\triangle_{p}$ of $x$ to $y$, with respect to the convex continuous function $f: E \rightarrow \mathbb{R}$ given by $f(x)=\frac{1}{p}\|x\|^{p}$, is defined as

$$
\triangle_{p}(x, y)=\frac{1}{q}\|x\|^{p}-\left\langle J^{p}(x), y\right\rangle+\frac{1}{p}\|y\|^{p},
$$

for all $x, y \in E$ and $p, q \in(1, \infty)$ such that $\frac{1}{p}+\frac{1}{q}=1$. 
Definition 1.3. Let $E$ be a smooth Banach space and $E^{*}$ its dual, the bifunctional $V_{p}$ with respect to the convex continuous function $f: E \rightarrow \mathbb{R}$ given by $f(x)=\frac{1}{p}\|x\|^{p}$, is defined by

$$
V_{p}\left(x^{*}, x\right)=\frac{1}{q}\left\|x^{*}\right\|^{q}-\left\langle x^{*}, x\right\rangle+\frac{1}{p}\|x\|^{p},
$$

for all $x \in E, x^{*} \in E^{*}$ and $p, q \in(1, \infty)$ such that $\frac{1}{p}+\frac{1}{q}=1$.

Definition 1.4. A Banach space $E$ is said to be uniformly convex, if for $x, y \in E, 0<\delta_{E}(\epsilon) \leq 1$, where $\delta_{E}(\epsilon)=\inf \left\{1-\left\|\frac{1}{2}(x+y)\right\| ;\|x\|=\|y\|=1,\|x-y\| \geq \epsilon\right.$, where $\left.0 \leq \epsilon \leq 2\right\}$.

Definition 1.5 ([19]). A Banach space $E$ is said to be uniformly smooth, if for $x, y \in E$ and $r>0$, $\lim _{r \rightarrow 0}\left(\frac{\rho_{E}(r)}{r}\right)=0$ where $\rho_{E}(r)=\frac{1}{2} \sup \{\|x+y\|+\|x-y\|-2:\|x\|=1,\|y\| \leq r\}$. Moreover,

(1) $\rho_{E}$ is continuous, convex and nondecreasing with $\rho_{E}(0)=0$ and $\rho_{E}(r) \leq r$.

(2) The function $r \mapsto \frac{\rho_{E}(r)}{r}$ is nondecreasing and fulfills $\frac{\rho_{E}(r)}{r}>0$ for all $r>0$.

Lemma 1.6 ([19]). Let $\left\{x_{n}\right\}$ be a sequence in a smooth Banach space E. Consider the following assertions;

(1) $\lim _{n \rightarrow \infty}\left\|x_{n}-x\right\|=0$

(2) $\lim _{n \rightarrow \infty}\left\|x_{n}\right\|=\|x\|$ and $\lim _{n \rightarrow \infty}\left\langle J^{p}\left(x_{n}\right), x\right\rangle=\left\langle J^{p}(x), x\right\rangle$

(3) $\lim _{n \rightarrow \infty} \triangle_{p}\left(x_{n}, x\right)=0$.

The assertions (1) $\Longrightarrow(2) \Longrightarrow(3)$ are valid. If $E$ is also uniformly convex, then the assertions are equivalent.

Lemma 1.7. Let $E$ be a reflexive and smooth Banach space and $E^{*}$ its dual. Let $\triangle_{p}$ and $V_{p}$ be the mappings defined as above and $J_{E}^{p}$ the generalized duality map on $E$. Then $\triangle_{p}(x, y)=V_{p}\left(J_{E}^{p} x, y\right)$ for all $x, y \in E$.

Proof. For $p, q \in(1, \infty)$ let $J_{E^{*}}^{q}: E^{*} \rightarrow E$ and $J_{E}^{p}: E \rightarrow E^{*}$ be duality mappings, where $J_{E^{*}}^{q} J_{E}^{p}=I$. It follows from $\frac{1}{p}+\frac{1}{q}=1$ that $p(q-1)=q$. So, we have that

$$
\begin{aligned}
\triangle_{p}(x, y) & =\frac{1}{q}\|x\|^{p}-\left\langle J_{E}^{p} x, y\right\rangle+\frac{1}{p}\|y\|^{p} \\
& =\frac{1}{q}\left\|J_{E^{*}}^{q} J_{E}^{p} x\right\|^{p}-\left\langle J_{E}^{p} x, y\right\rangle+\frac{1}{p}\|y\|^{p} \\
& =\frac{1}{q}\left\|J_{E}^{p} x\right\|^{p(q-1)}-\left\langle J_{E}^{p} x, y\right\rangle+\frac{1}{p}\|y\|^{p} \\
& =\frac{1}{q}\left\|J_{E}^{p} x\right\|^{q}-\left\langle J_{E}^{p} x, y\right\rangle+\frac{1}{p}\|y\|^{p} \\
& =V_{p}\left(J_{E}^{p} x, y\right) .
\end{aligned}
$$

Lemma $1.8([19])$. Let $E$ be a reflexive, strictly convex and smooth Banach space and $J^{p}$ be the duality mapping of $E$. Then

(i) for every closed and convex subset $C \subset E$ and $x \in E$, there exists a unique element $\Pi_{C}^{p}(x) \in C$ such that $\triangle_{p}\left(x, \Pi_{C}^{p}(x)\right)=\min _{y \in C} \triangle_{p}(x, y) ; \Pi_{C}^{p}(x)$ is called the Bregman projection of $x$ onto $C$, with respect to the function $f(x)=\frac{1}{p}\|x\|^{p}$. Moreover, $x_{0} \in C$ is the Bregman projection of $x$ onto $C$ if

$$
\left\langle J^{p}\left(x_{0}-x\right), y-x_{0}\right\rangle \geq 0
$$

or equivalently

$$
\triangle_{p}\left(x_{0}, y\right) \leq \triangle_{p}(x, y)-\triangle_{p}\left(x, x_{0}\right) \text { for every } y \in C .
$$


(ii) the Bregman projection and the metric projection are related via $P_{C}(x)-x=\Pi_{C-x}^{p}(0), \forall x \in E$. Especially, we have $P_{C}(0)=\Pi_{C}^{p}(0)$ and thus $\left\|\Pi_{C}^{p}(0)\right\|=\min _{y \in C}\|y\|$.

The uniform convexity of $E$ implies that $E$ is reflexive and $E^{*}$ is uniformly smooth. Therefore, Theorem 2 in [27], for $x, y \in E$ and $x^{*}, y^{*} \in E^{*}$ and $\|x+y\|^{p}$ replaced by $\left\|x^{*}-y^{*}\right\|^{q}$ gives the following technical result.

Lemma 1.9. For the uniformly smooth space $E^{*}$, with the duality map $J^{q}, \forall x^{*}, y^{*} \in E^{*}$, we have

$$
\begin{aligned}
\left\|x^{*}-y^{*}\right\|^{q} & \leq\left\|x^{*}\right\|^{q}-q\left\langle J^{q}\left(x^{*}\right), y^{*}\right\rangle+\overline{\sigma_{q}}\left(x^{*}, y^{*}\right) \text { where } \\
\bar{\sigma}_{q}\left(x^{*}, y^{*}\right) & =q G_{q} \int_{0}^{1} \frac{\left(\left\|x^{*}-t y^{*}\right\| \vee\left\|x^{*}\right\|\right)^{q}}{t} \rho_{E^{*}}\left(\frac{t\left\|y^{*}\right\|}{2\left(\left\|x^{*}-t y^{*}\right\| \vee\left\|x^{*}\right\|\right)}\right) \mathrm{d} t \\
\text { and } G_{q} & =8 \vee 64 c K_{q}^{-1} \text { with } c, K_{q}>0 .
\end{aligned}
$$

Lemma 1.10 ([19]). Let $E$ be a reflexive, strictly convex and smooth Banach space. We write $\triangle_{q}^{*}(x, y)=\frac{1}{p}\left\|x^{*}\right\|^{q}-\left\langle J_{E^{*}}^{q} x^{*}, y^{*}\right\rangle+\frac{1}{q}\left\|y^{*}\right\|^{q}$ for $x^{*}=J_{E}^{p}(x), y^{*}=J_{E}^{p}(y)$ for the Bregman distance on the dual space $E^{*}$ with respect to the function $f_{q}^{*}\left(x^{*}\right)=\frac{1}{q}\left\|x^{*}\right\|^{q}$. Then we have $\triangle_{p}(x, y)=\triangle_{q}^{*}\left(x^{*}, y^{*}\right)$.

Lemma 1.11. Let $E$ be a reflexive, smooth and strictly convex Banach space. Then for all $x, y, z \in E$ and $x^{*}=J_{E}^{p} x, z^{*}=J_{E}^{p} z$, the following hold:

(1) $\triangle_{p}(x, y) \geq 0$ and $\triangle_{p}(x, y)=0$ if $x=y$;

(2) $\triangle_{p}(x, y)=\triangle_{p}(x, z)+\triangle_{p}(z, y)+\left\langle x^{*}-z^{*}, z-y\right\rangle$.

Proof. The property (1) is proved in [19]. For (2) we have that

$$
\begin{aligned}
\triangle_{p}(x, z)+\triangle_{p}(z, y) & =\frac{1}{q}\|x\|^{p}-\left\langle x^{*}, z\right\rangle+\frac{1}{p}\|z\|^{p}+\frac{1}{q}\|z\|^{p}-\left\langle z^{*}, y\right\rangle+\frac{1}{p}\|y\|^{p} \\
& =\frac{1}{q}\|x\|^{p}-\left\langle x^{*}, z\right\rangle+\|z\|^{p}-\left\langle z^{*}, y\right\rangle+\frac{1}{p}\|y\|^{p}+\left\langle x^{*}, y\right\rangle-\left\langle x^{*}, y\right\rangle \\
& =\left(\frac{1}{q}\|x\|^{p}-\left\langle x^{*}, y\right\rangle+\frac{1}{p}\|y\|^{p}\right)+\left\langle z^{*}, z\right\rangle-\left\langle z^{*}, y\right\rangle+\left\langle x^{*}, y\right\rangle-\left\langle x^{*}, z\right\rangle \\
& \Leftrightarrow \triangle_{p}(x, y)=\triangle_{p}(x, z)+\triangle_{p}(z, y)+\left\langle x^{*}-z^{*}, z-y\right\rangle .
\end{aligned}
$$

If $E$ is smooth and $f(x)=\frac{1}{p}\|x\|^{p}$, then the following result holds (cf. Prop. 5 in [18]).

Lemma 1.12. Let $E$ be a smooth Banach space and $f: E \rightarrow R$ be a continuous convex function given by $f(x)=\frac{1}{p}\|x\|^{p}$. If $x_{0} \in E$ and the sequence $\left\{\triangle_{p}\left(x_{n}, x_{0}\right)\right\}_{n=1}^{\infty}$ is bounded, then the sequence $\left\{x_{n}\right\}$ is also bounded.

\section{MAIN RESUltS}

Let $E_{1}$ and $E_{2}$ be uniformly convex and uniformly smooth Banach spaces and $E_{1}^{*}$ and $E_{2}^{*}$ be their duals, respectively. Let $U: E_{1} \rightarrow 2^{E_{1}^{*}}$ and $T: E_{2} \rightarrow 2^{E_{2}^{*}}$ be multi-valued maximal monotone operators. For $K \subset E_{1}$, closed and convex, $\delta>0$ and $p, q \in(1, \infty)$, let $A: E_{1} \rightarrow E_{2}$ be a bounded and linear operator, $A^{*}$ denotes the adjoint of $A$ and $A K$ be closed and convex. Suppose that $\Pi_{A K}^{p}: E_{2} \rightarrow A K$ is the Bregman projection onto a closed and convex subset $A K$. Let $B_{\delta}^{U}: E_{1} \rightarrow E_{1}$ be the generalized resolvent operator defined by $B_{\delta}^{U}=\left(J_{E_{1}}^{p}+\delta U\right)^{-1} J_{E_{1}}^{p}$ and $B_{\delta}^{T}: E_{2} \rightarrow E_{2}$ be another generalized resolvent operator defined by $B_{\delta}^{T}=$ $\left(J_{E_{2}}^{p}+\delta T\right)^{-1} J_{E_{2}}^{p}$. Let us denote the solutions of variational inclusion problem with respect to $U$ and $T$ by $S O L V I P(U)$ and $S O L V I P(T)$, respectively. Let the set of solutions of split variational inclusion problem be 
given by $\Omega=\left\{x^{*} \in \operatorname{SOLVIP}(U) ; A x^{*} \in \operatorname{SOLVIP}(T)\right\} \neq \emptyset$. Let $x_{1} \in E_{1}$ be chosen arbitrarily and the sequence $\left\{x_{n}\right\} \subset E_{1}$ be defined as follows;

$$
\left\{\begin{array}{l}
u_{n}=B_{\delta_{n}}^{U}\left(J_{E_{1}^{*}}^{q}\left(J_{E_{1}}^{p} x_{n}-\lambda_{n} A^{*} J_{E_{2}}^{p}\left(I-\Pi_{A K}^{p} B_{\delta_{n}}^{T}\right) A x_{n}\right)\right) \\
K_{n+1}=\left\{v \in K_{n}: \triangle_{p}\left(u_{n}, v\right) \leq \triangle_{p}\left(x_{n}, v\right)\right\} \\
x_{n+1}=\Pi_{K_{n+1}}^{p}\left(x_{1}\right), n \geq 1
\end{array}\right.
$$

where $\delta_{n} \in(0, \infty)$. It is remarked that we have replaced the gradient algorithm in (1.1) [the projection maps in (1.3), respectively] with the resolvent operators and used the generalized duality map in our algorithm.

We shall strictly employ the above terminology in the sequel.

Lemma 2.1. Suppose that $\bar{\sigma}_{q}$ is the function in (1.4) for the characteristic inequality of the uniformly smooth space $E_{1}^{*}$. For the sequence $\left\{x_{n}\right\} \subset E_{1}$ defined by (2.1), let $0 \neq x_{n} \in E_{1}, 0 \neq A$ and $0 \neq J_{E_{2}}^{p}\left(I-\Pi_{A K}^{p} B_{\delta_{n}}^{T}\right) A x_{n} \in E_{2}^{*}$. Let $\lambda_{n}>0$ and $\mu_{n}>0$ be defined, respectively, by

$$
\lambda_{n}=\frac{1}{\|A\|} \frac{1}{\left\|J_{E_{2}}^{p}\left(I-\Pi_{A K}^{p} B_{\delta_{n}}^{T}\right) A x_{n}\right\|} \text { and } \mu_{n}=\frac{1}{\left\|x_{n}\right\|^{p-1}} .
$$

Then

$$
\frac{1}{q} \overline{\sigma_{q}}\left(J_{E_{1}}^{p} x_{n}, \lambda_{n} A^{*} J_{E_{2}}^{p}\left(I-\Pi_{A K}^{p} B_{\delta_{n}}^{T}\right) A x_{n}\right) \leq \begin{cases}2^{q} G_{q}\left\|J_{E_{1}}^{p} x_{n}\right\|^{q} \rho_{E_{1}^{*}}\left(\mu_{n}\right) & \text { if } \mu_{n} \in(0,1], \\ 2^{q} G_{q} \rho_{E_{1}^{*}}\left(\mu_{n}\right) & \text { if } \mu_{n} \in(1, \infty),\end{cases}
$$

where $G_{q}$ is the constant defined in Lemma 1.9 and $\rho_{E_{1}^{*}}$ is the modulus of smoothness of $E_{1}^{*}$.

Proof. By Lemma 1.9, we have

$$
\begin{aligned}
\frac{1}{q} \bar{\sigma}_{q}\left(J_{E_{1}}^{p} x_{n}, \lambda_{n} A^{*} J_{E_{2}}^{p}\left(I-\Pi_{A K}^{p} B_{\delta_{n}}^{T}\right) A x_{n}\right)= & G_{q} \int_{0}^{1} \frac{\left(\left\|J_{E_{1}}^{p} x_{n}-\lambda_{n} A^{*} J_{E_{2}}^{p}\left(I-\Pi_{A K}^{p} B_{\delta_{n}}^{T}\right) A x_{n}\right\| \vee\left\|J_{E_{1}}^{p} x_{n}\right\|\right)^{q}}{t} \\
& \times \rho_{E^{*}}\left(\frac{t\left\|\lambda_{n} A^{*} J_{E_{2}}^{p}\left(I-\Pi_{A K}^{p} B_{\delta_{n}}^{T}\right) A x_{n}\right\|}{\left(\left\|J_{E_{1}}^{p} x_{n}-\lambda_{n} A^{*} J_{E_{2}}^{p}\left(I-\Pi_{A K}^{p} B_{\delta_{n}}^{T}\right) A x_{n}\right\| \vee\left\|J_{E_{1}}^{p} x_{n}\right\|\right)}\right) \mathrm{d} t
\end{aligned}
$$

for every $t \in[0,1]$.

We note that

$$
\left\|J_{E_{1}}^{p} x_{n}-\lambda_{n, i} A^{*} J_{E_{2}}^{p}\left(I-\Pi_{A K}^{p} B_{\delta_{n}}^{T}\right) A x_{n}\right\| \leq\left\|x_{n}\right\|^{p-1}+\left\|\lambda_{n} A^{*} J_{E_{2}}^{p}\left(I-\Pi_{A K}^{p} B_{\delta_{n}}^{T}\right) A x_{n}\right\| .
$$

By (2.2), with $x_{n} \neq 0$

$$
\lambda_{n}=\frac{\mu_{n}}{\|A\|} \frac{\left\|x_{n}\right\|^{p-1}}{\left\|J_{E_{2}}^{p}\left(I-\Pi_{A K}^{p} B_{\delta_{n}}^{T}\right) A x_{n}\right\|}
$$

and so we have that

$$
\left\|J_{E_{1}}^{p} x_{n}-\lambda_{n} A^{*} J_{E_{2}}^{p}\left(I-\Pi_{A K}^{p} B_{\delta_{n}}^{T}\right) A x_{n}\right\| \leq\left(1+\mu_{n}\right)\left\|x_{n}\right\|^{p-1}
$$

and

$$
\begin{cases}\left\|x_{n}\right\|^{p-1} \leq\left\|J_{E_{1}}^{p} x_{n}-\lambda_{n} A^{*} J_{E_{2}}^{p}\left(I-\Pi_{A K}^{p} B_{\delta_{n}}^{T}\right) A x_{n}\right\| \vee\left\|J_{E_{1}}^{p} x_{n}\right\| \leq 2\left\|x_{n}\right\|^{p-1} & \text { if } \mu_{n} \in(0,1] \\ \left\|x_{n}\right\|^{p-1} \leq\left\|J_{E_{1}}^{p} x_{n}-\lambda_{n} A^{*} J_{E_{2}}^{p}\left(I-\Pi_{A K}^{p} B_{\delta_{n}}^{T}\right) A x_{n}\right\| \vee\left\|J_{E_{1}}^{p} x_{n}\right\| \leq 2 & \text { if } \mu_{n} \in(1, \infty) .\end{cases}
$$


By (2.6), (2.5) and Definition 1.5(2), we get

$$
\begin{aligned}
\rho_{E_{1}^{*}}\left(\frac{t\left\|\lambda_{n} A^{*} J_{E_{2}}^{p}\left(I-\Pi_{A K}^{p} B_{\delta_{n}}^{T}\right) A x_{n}\right\|}{\left(\left\|J_{E_{1}}^{p} x_{n}-t \lambda_{n} A^{*} J_{E_{2}}^{p}\left(I-\Pi_{A K}^{p} B_{\delta_{n}}^{T}\right) A x_{n}\right\| \vee\left\|J_{E_{1}}^{p} x_{n}\right\|\right)}\right) & \leq \rho_{E_{1}^{*}}\left(\frac{t\left\|\lambda_{n} A^{*} J_{E_{2}}^{p}\left(I-\Pi_{A K}^{p} B_{\delta_{n}}^{T}\right) A x_{n}\right\|}{\left\|x_{n}\right\|^{p-1}}\right) \\
& =\rho_{E_{1}^{*}}\left(t \mu_{n}\right) .
\end{aligned}
$$

Substituting (2.7) and (2.6) into (2.4), and using nondecreasingness of $\rho_{E_{1}^{*}}$, we get (2.3) as required.

Lemma 2.2. For the sequence $\left\{x_{n}\right\} \subset E_{1}$ defined by (2.1), let $0 \neq x_{n}, 0 \neq J_{E_{2}}^{p}\left(I-\Pi_{A K}^{p} B_{\delta_{n}}^{T}\right) A x_{n} \in E_{2}^{*}$, and $\lambda_{n}>0$ and $\mu_{n}>0$ be defined by (2.2) and $\lambda_{n}$ and $\mu_{n}$ are chosen such that

$$
\rho_{E_{1}^{*}}\left(\mu_{n}\right)= \begin{cases}\frac{\iota}{2^{q} G_{q}\|A\|} \times \frac{\left\langle J_{E_{2}}^{p}\left(I-\Pi_{A K}^{p} B_{\delta_{n}}^{T}\right) A x_{n},\left(I-\Pi_{A K}^{p} B_{\delta_{n}}^{T}\right) A x_{n}\right\rangle}{\left\|J_{E_{1}}^{p} x_{n}\right\| q\left\|J_{E_{2}}^{p}\left(I-\Pi_{A K}^{p} B_{\delta_{n}}^{T}\right) A x_{n}\right\|}, & \text { if } \mu_{n} \in(0,1], \\ \frac{\iota}{2^{q} G_{q}\|A\|} \times \frac{\left\langle J_{E_{2}}^{p}\left(I-\Pi_{A K}^{p} B_{\delta_{n}}^{T}\right) A x_{n},\left(I-\Pi_{A K}^{p} B_{\delta_{n}}^{T}\right) A x_{n}\right\rangle}{\left\|J_{E_{2}}^{p}\left(I-\Pi_{A K}^{p} B_{\delta_{n}}^{T}\right) A x_{n}\right\|}, & \text { if } \mu_{n} \in(1, \infty),\end{cases}
$$

where $\iota \in(0,1)$. Then, for all $v \in \Omega$, we get

$$
\triangle_{p}\left(u_{n}, v\right) \leq \triangle_{p}\left(x_{n}, v\right)-[1-\iota] \frac{\left\langle J_{E_{2}}^{p}\left(I-\Pi_{A K}^{p} B_{\delta_{n}}^{T}\right) A x_{n},\left(I-\Pi_{A K}^{p} B_{\delta_{n}}^{T}\right) A x_{n}\right\rangle}{\|A\|\left\|J_{E_{2}}^{p}\left(I-\Pi_{A K}^{p} B_{\delta_{n}}^{T}\right) A x_{n}\right\|} .
$$

Proof. For $v=B_{\gamma}^{U} v$ and $A v=B_{\gamma}^{T} A v$, by Lemma 1.7, we have that

$$
\begin{aligned}
\triangle_{p}\left(u_{n}, v\right)= & \triangle_{p}\left(B_{\delta_{n}}^{U}\left(J_{E_{1}^{*}}^{q}\left(J_{E_{1}}^{p} x_{n}-\lambda_{n} A^{*} J_{E_{2}}^{p}\left(I-\Pi_{A K}^{p} B_{\delta_{n}}^{T}\right) A x_{n}\right)\right), v\right) \\
= & \triangle_{p}\left(B_{\delta_{n}}^{U}\left(J_{E_{1}^{*}}^{q}\left(J_{E_{1}}^{p} x_{n}-\lambda_{n} A^{*} J_{E_{2}}^{p}\left(I-\Pi_{A K}^{p} B_{\delta_{n}}^{T}\right) A x_{n}\right)\right), B_{\delta_{n}}^{U} v\right) \\
\leq & V_{p}\left(J_{E_{1}}^{p} x_{n}-\lambda_{n} A^{*} J_{E_{2}}^{p}\left(I-\Pi_{A K}^{p} B_{\delta_{n}}^{T}\right) A x_{n}, v\right) \\
= & \frac{1}{q}\left\|J_{E_{1}}^{p} x_{n}-\lambda_{n} A^{*} J_{E_{2}}^{p}\left(I-\Pi_{A K}^{p} B_{\delta_{n}}^{T}\right) A x_{n}\right\|^{q}+\frac{1}{p}\|v\|^{p} \\
& -\left\langle J_{E_{1}}^{p} x_{n}, v\right\rangle+\left\langle\lambda_{n} A^{*} J_{E_{2}}^{p}\left(I-\Pi_{A K}^{p} B_{\delta_{n}}^{T}\right) A x_{n}, v\right\rangle,
\end{aligned}
$$

where,

$$
\begin{aligned}
\left\langle\lambda_{n} A^{*} J_{E_{2}}^{p}\left(I-\Pi_{A K}^{p} B_{\delta_{n}}^{T}\right) A x_{n}, v\right\rangle= & \left\langle\lambda_{n} J_{E_{2}}^{p}\left(I-\Pi_{A K}^{p} B_{\delta_{n}}^{T}\right) A x_{n}, A v-A x_{n}+A x_{n}-\Pi_{A K}^{p} B_{\delta_{n}}^{T} A x_{n}\right\rangle \\
& +\left\langle\lambda_{n} J_{E_{2}}^{p}\left(I-\Pi_{A K}^{p} B_{\delta_{n}}^{T}\right) A x_{n}, \Pi_{A K}^{p} B_{\delta_{n}}^{T} A x_{n}-A x_{n}+A x_{n}\right\rangle \\
= & -\left\langle\lambda_{n} J_{E_{2}}^{p}\left(\Pi_{A K}^{p} B_{\delta_{n}}^{T}-I\right) A x_{n},\left(A v-A x_{n}\right)-\left(\Pi_{A K}^{p} B_{\delta_{n}}^{T}-I\right) A x_{n}\right\rangle \\
& -\left\langle\lambda_{n} J_{E_{2}}^{p}\left(I-\Pi_{A K}^{p} B_{\delta_{n}}^{T}\right) A x_{n},\left(I-\Pi_{A K}^{p} B_{\delta_{n}}^{T}\right) A x_{n}\right\rangle \\
+ & \left\langle\lambda_{n} J_{E_{2}}^{p}\left(I-\Pi_{A K}^{p} B_{\delta_{n}}^{T}\right) A x_{n}, A x_{n}\right\rangle .
\end{aligned}
$$

As $A K$ is closed and convex so by Lemma 1.8(i) and the variational inequality for the Bregman projection of zero onto $A K-A x_{n}$, as in Lemma 1.8(ii), we arrive at

$$
\left\langle\lambda_{n} J_{E_{2}}^{p}\left(\Pi_{A K}^{p} B_{\delta_{n}}^{T}-I\right) A x_{n},\left(A v-A x_{n}\right)-\left(\Pi_{A K}^{p} B_{\delta_{n}}^{T}-I\right) A x_{n}\right\rangle \geq 0
$$

and therefore, we obtain

$$
\begin{aligned}
\left\langle\lambda_{n} A^{*} J_{E_{2}}^{p}\left(I-\Pi_{A K}^{p} B_{\delta_{n}}^{T}\right) A x_{n}, v\right\rangle \leq & -\left\langle\lambda_{n} J_{E_{2}}^{p}\left(I-\Pi_{A K}^{p} B_{\delta_{n}}^{T}\right) A x_{n},\left(I-\Pi_{A K}^{p} B_{\delta_{n}}^{T}\right) A x_{n}\right\rangle \\
& +\left\langle\lambda_{n} J_{E_{2}}^{p}\left(I-\Pi_{\Gamma}^{p} B_{\delta_{n}}^{T}\right) A x_{n}, A x_{n}\right\rangle .
\end{aligned}
$$


In addition, by Lemma 1.9, we have that

$$
\begin{aligned}
\frac{1}{q}\left\|J_{E_{1}}^{p} x_{n}-\lambda_{n} A^{*} J_{E_{2}}^{p}\left(I-\Pi_{A K}^{p} B_{\delta_{n}}^{T}\right) A x_{n}\right\|^{q} \leq & \frac{1}{q}\left\|J_{E_{1}}^{p} x_{n}\right\|^{q}-\lambda_{n}\left\langle A x_{n}, J_{E_{2}}^{p}\left(I-\Pi_{A K}^{p} B_{\delta_{n}}^{T}\right) A x_{n}\right\rangle \\
& +\frac{1}{q} \overline{\sigma_{q}}\left(J_{E_{1}}^{p} x_{n}, \lambda_{n} A^{*} J_{E_{2}}^{p}\left(I-\Pi_{A K}^{p} B_{\delta_{n}}^{T}\right) A x_{n}\right)
\end{aligned}
$$

By Lemma 2.1 and (2.12), we have that

$$
\begin{aligned}
\frac{1}{q}\left\|J_{E_{1}}^{p} x_{n}-\lambda_{n} A^{*} J_{E_{2}}^{p}\left(I-\Pi_{A K}^{p} B_{\delta_{n}}^{T}\right) A x_{n}\right\|^{q} \leq & \frac{1}{q}\left\|J_{E_{1}}^{p} x_{n}\right\|^{q}-\lambda_{n}\left\langle A x_{n}, J_{E_{2}}^{p}\left(I-\Pi_{A K}^{p} B_{\delta_{n}}^{T}\right) A x_{n}\right\rangle \\
& +2^{q} G_{q}\left\|J_{E_{1}}^{p} x_{n}\right\|^{q} \rho_{E_{1}^{*}}\left(\mu_{n}\right) .
\end{aligned}
$$

Substituting (2.13) and (2.11) into (2.10), we have that

$$
\begin{aligned}
\triangle_{p}\left(u_{n}, v\right) \leq & \frac{1}{q}\left\|J_{E_{1}}^{p} x_{n}\right\|^{q}+\frac{1}{p}\|v\|^{p}-\left\langle J_{E_{1}}^{p} x_{n}, v\right\rangle+2^{q} G_{q}\left\|J_{E_{1}}^{p} x_{n}\right\|^{q} \rho_{E_{1}^{*}}\left(\mu_{n}\right) \\
& -\left\langle\lambda_{n} J_{E_{2}}^{p}\left(I-\Pi_{A K}^{p} B_{\delta_{n}}^{T}\right) A x_{n},\left(I-\Pi_{A K}^{p} B_{\delta_{n}}^{T}\right) A x_{n}\right\rangle \\
= & \triangle_{p}\left(x_{n}, v\right)+2^{q} G_{q}\left\|J_{E_{1}}^{p} x_{n}\right\|^{q} \rho_{E_{1}^{*}}\left(\mu_{n}\right) \\
& -\left\langle\lambda_{n} J_{E_{2}}^{p}\left(I-\Pi_{A K}^{p} B_{\delta_{n}}^{T}\right) A x_{n},\left(I-\Pi_{A K}^{p} B_{\delta_{n}}^{T}\right) A x_{n}\right\rangle .
\end{aligned}
$$

Substituting (2.2) and (2.8) into (2.14), we have that

$$
\begin{aligned}
\triangle_{p}\left(u_{n}, v\right) \leq & \triangle_{p}\left(x_{n}, v\right)+\frac{\iota\left\langle\left(J_{E_{2}}^{p}\left(I-\Pi_{A K}^{p} B_{\delta_{n}}^{T}\right) A x_{n},\left(I-\Pi_{A K}^{p} B_{\delta_{n}}^{T}\right) A x_{n}\right\rangle\right.}{\|A\|\left\|J_{E_{2}}^{p}\left(I-\Pi_{A K}^{p} B_{\delta_{n}}^{T}\right) A u_{n}\right\|} \\
& -\frac{\left\langle\left(J_{E_{2}}^{p}\left(I-\Pi_{A K}^{p} B_{\delta_{n}}^{T}\right) A x_{n},\left(I-\Pi_{A K}^{p} B_{\delta_{n}}^{T}\right) A x_{n}\right\rangle\right.}{\|A\|\left\|J_{E_{2}}^{p}\left(I-\Pi_{A K}^{p} B_{\delta_{n}}^{T}\right) A x_{n}\right\|} \\
= & \triangle_{p}\left(x_{n}, v\right)-[1-\iota] \frac{\left\langle J_{E_{2}}^{p}\left(I-\Pi_{A K}^{p} B_{\delta_{n}}^{T}\right) A x_{n},\left(I-\Pi_{A K}^{p} B_{\delta_{n}}^{T}\right) A x_{n}\right\rangle}{\|A\|\left\|J_{E_{2}}^{p}\left(I-\Pi_{A K}^{p} B_{\delta_{n}}^{T}\right) A x_{n}\right\|} .
\end{aligned}
$$

Thus, (2.9) holds.

We now prove our main result.

Theorem 2.3. For $\delta>0$ and $p, q \in(1, \infty)$, let $\left(I-\Pi_{A K}^{p} B_{\delta}^{T}\right)$ be demiclosed at zero. Let $x_{1} \in E_{1}$ be chosen arbitrarily and the sequence $\left\{x_{n}\right\}$ be defined by (2.1), where

$$
\lambda_{n}=\left\{\begin{array}{ll}
\frac{1}{\|A\|} \frac{1}{\left\|J_{E_{2}}^{p}\left(I-\Pi_{A K}^{p} B_{\delta_{n}}^{T}\right) A x_{n}\right\|}, & x_{n} \neq 0 \\
\frac{1}{\|A\|^{p}} \frac{\left\langle J_{E_{2}}^{p}\left(I-\Pi_{A K}^{p} B_{\delta_{n}}^{T}\right) A x_{n},\left(I-\Pi_{A K}^{p} B_{\delta_{n}}^{T}\right) A x_{n}\right\rangle^{p-1}}{\left\|J_{E_{2}}^{p}\left(I-\Pi_{A K}^{p} B_{\delta_{n}}^{T}\right) A x_{n}\right\|^{p}}, & x_{n}=0
\end{array} \text { and } \mu_{n}=\frac{1}{\left\|x_{n}\right\|^{p-1}}\right.
$$

are chosen such that equation (2.8) holds. If $\Omega=\left\{x^{*} \in \operatorname{SOLVIP}(U) ; A x^{*} \in \operatorname{SOLVIP}(T)\right\} \neq \emptyset$, then $\left\{x_{n}\right\}$ converges strongly to $x^{*} \in \Omega$, where $\Pi_{A K}^{p} B_{\delta_{n}}^{T}\left(A x^{*}\right)=B_{\delta_{n}}^{T}\left(A x^{*}\right)$.

Proof. We will divide the proof into two steps.

Step one. We show that $\left\{x_{n}\right\}$ is a bounded sequence.

Assume that $\left\|J_{E_{2}}^{p}\left(I-\Pi_{A K}^{p} B_{\delta_{n}}^{T}\right) A x_{n}\right\|=0$. Then from $v=B_{\gamma}^{U} v$, Lemma 1.7 and $v \in \Omega$, we get

$$
\triangle_{p}\left(u_{n}, v\right)=\triangle_{p}\left(B_{\delta_{n}}^{U}\left(J_{E_{1}^{*}}^{q}\left(J_{E_{1}}^{p} x_{n}\right)\right), B_{\delta_{n}}^{U} v\right) \leq V_{p}\left(J_{E_{1}}^{p} x_{n}, v\right)=\triangle_{p}\left(x_{n}, v\right) .
$$


Next assume that $\left\|J_{E_{2}}^{p}\left(I-\Pi_{A K}^{p} B_{\delta_{n}}^{T}\right) A x_{n}\right\| \neq 0$ and $x_{n} \neq 0$. Then for $v \in \Omega$, by Lemma 2.2 , we get

$$
\begin{aligned}
\triangle_{p}\left(u_{n}, v\right) & \leq \triangle_{p}\left(x_{n}, v\right)-[1-\iota] \frac{\left\langle J_{E_{2}}^{p}\left(I-\Pi_{A K}^{p} B_{\delta_{n}}^{T}\right) A x_{n},\left(I-\Pi_{A K}^{p} B_{\delta_{n}}^{T}\right) A x_{n}\right\rangle}{\|A\|\left\|J_{E_{2}}^{p}\left(I-\Pi_{A K}^{p} B_{\delta_{n}}^{T}\right) A x_{n}\right\|} \\
& \leq \triangle_{p}\left(x_{n}, v\right) .
\end{aligned}
$$

For $x_{n}=0$, we have

$$
\triangle_{p}\left(x_{n}, v\right)=\frac{1}{p}\|v\|^{p}
$$

and so by (2.19), we have that

$$
\begin{aligned}
\triangle_{p}\left(u_{n}, v\right)= & \frac{1}{q}\left\|\lambda_{n} A^{*} J_{E_{2}}^{p}\left(I-\Pi_{A K}^{p} B_{\delta_{n}}^{T}\right) A x_{n}\right\|^{q} \\
& +\triangle_{p}\left(x_{n}, v\right)+\lambda_{n}\left\langle J_{E_{2}}^{p}\left(I-\Pi_{A K}^{p} B_{\delta_{n}}^{T}\right) A x_{n}, A v\right\rangle .
\end{aligned}
$$

Substituting (2.11) in (2.20), we have that

$$
\begin{aligned}
\triangle_{p}\left(u_{n}, v\right) \leq & \frac{1}{q}\left\|\lambda_{n} A^{*} J_{E_{2}}^{p}\left(I-\Pi_{A K}^{p} B_{\delta_{n}}^{T}\right) A x_{n}\right\|^{q} \\
& +\triangle_{p}\left(x_{n}, v\right)+\lambda_{n}\left\langle J_{E_{2}}^{p}\left(I-\Pi_{A K}^{p} B_{\delta_{n}}^{T}\right) A x_{n}, A x_{n}\right\rangle \\
& -\lambda_{n}\left\langle J_{E_{2}}^{p}\left(I-\Pi_{A K}^{p} B_{\delta_{n}}^{T}\right) A x_{n},\left(I-\Pi_{A K}^{p} B_{\delta_{n}}^{T}\right) A x_{n}\right\rangle .
\end{aligned}
$$

By (2.15), we have that

$$
\frac{1}{q}\left\|\lambda_{n} A^{*} J_{E_{2}}^{p}\left(I-\Pi_{A K}^{p} B_{\delta_{n}}^{T}\right) A x_{n}\right\|^{q}=\frac{1}{q} \frac{1}{\|A\|^{p}} \frac{\left\langle J_{E_{2}}^{p}\left(I-\Pi_{A K}^{p} B_{\delta_{n}}^{T}\right) A x_{n},\left(I-\Pi_{A K}^{p} B_{\delta_{n}}^{T}\right) A x_{n}\right\rangle^{p}}{\left\|J_{E_{2}}^{p}\left(I-\Pi_{A K}^{p} B_{\delta_{n}}^{T}\right) A x_{n}\right\|^{p}} .
$$

Substituting (2.22) into (2.21), we have that

$$
\begin{aligned}
\triangle_{p}\left(u_{n}, v\right) \leq & \frac{1}{q} \| \frac{\left\langle J_{E_{2}}^{p}\left(I-\Pi_{A K}^{p} B_{\delta_{n}}^{T}\right) A x_{n},\left(I-\Pi_{A K}^{p} B_{\delta_{n}}^{T}\right) A x_{n}\right\rangle^{p}}{\left\|J_{E_{2}}^{p}\left(I-\Pi_{A K}^{p} B_{\delta_{n}}^{T}\right) A x_{n}\right\|^{p}} \\
& +\triangle_{p}\left(x_{n}, v\right)+\lambda_{n}\left\langle J_{E_{2}}^{p}\left(I-\Pi_{A K}^{p} B_{\delta_{n}}^{T}\right) A x_{n}, A x_{n}\right\rangle \\
& -\lambda_{n}\left\langle J_{E_{2}}^{p}\left(I-\Pi_{A K}^{p} B_{\delta_{n}}^{T}\right) A x_{n},\left(I-\Pi_{A K}^{p} B_{\delta_{n}}^{T}\right) A x_{n}\right\rangle \\
\leq & \left(1-\frac{1}{p}\right) \frac{1}{\|A\|^{p}} \frac{\left\langle J_{E_{2}}^{p}\left(I-\Pi_{A K}^{p} B_{\delta_{n}}^{T}\right) A x_{n},\left(I-\Pi_{A K}^{p} B_{\delta_{n}}^{T}\right) A x_{n}\right\rangle^{p}}{\left\|J_{E_{2}}^{p}\left(I-\Pi_{A K}^{p} B_{\delta_{n}}^{T}\right) A x_{n}\right\|^{p}} \\
& +\triangle_{p}\left(x_{n}, v\right)+\lambda_{n}\left\|J_{E_{2}}^{p}\left(I-\Pi_{A K}^{p} B_{\delta_{n}}^{T}\right) A x_{n}\right\|\left\|A x_{n}\right\| \\
& -\frac{1}{\|A\|^{p}} \frac{\left\langle J_{E_{2}}^{p}\left(I-\Pi_{A K}^{p} B_{\delta_{n}}^{T}\right) A x_{n},\left(I-\Pi_{A K}^{p} B_{\delta_{n}}^{T}\right) A x_{n}\right\rangle^{p}}{\left\|J_{E_{2}}^{p}\left(I-\Pi_{A K}^{p} B_{\delta_{n}}^{T}\right) A x_{n}\right\|^{p}} \\
= & \triangle_{p}\left(x_{n}, v\right)-\frac{1}{p\|A\|^{p}} \frac{\left\langle J_{E_{2}}^{p}\left(I-\Pi_{A K}^{p} B_{\delta_{n}}^{T}\right) A x_{n},\left(I-\Pi_{A K}^{p} B_{\delta_{n}}^{T}\right) A x_{n}\right\rangle^{p}}{\left\|J_{E_{2}}^{p}\left(I-\Pi_{A K}^{p} B_{\delta_{n}}^{T}\right) A x_{n}\right\|^{p}} .
\end{aligned}
$$

This implies that

$$
\triangle_{p}\left(u_{n}, v\right) \leq \triangle_{p}\left(x_{n}, v\right) .
$$

By (2.1), (2.16), (2.18) and (2.24), $v \in K_{n}$ so that $\Omega \subset K_{n}$. 
We know from (2.1), $x_{n}=\Pi_{K_{n}}^{p} x_{1}$. Then, by Lemma 1.8, we have

$$
\triangle_{p}\left(x_{n}, x_{1}\right)=\triangle_{p}\left(\Pi_{K}^{p} x_{1}, x_{1}\right) \leq \triangle_{p}\left(v, x_{1}\right)-\triangle_{p}\left(v, x_{n}\right) \Rightarrow \triangle_{p}\left(x_{n}, x_{1}\right) \leq \triangle_{p}\left(v, x_{1}\right) \forall v \in \Omega \subset K_{n} .
$$

By (2.25), the sequence $\left\{\triangle_{p}\left(x_{n}, x_{1}\right)\right\}$ is bounded and therefore by Lemma 1.12, $\left\{x_{n}\right\}$ is bounded. Hence, $\left\{u_{n}\right\}$ is also bounded. Consequently, there exists a subsequence $x_{n_{j}}$ such that $x_{n_{j}} \rightarrow x^{*}$ as $j \rightarrow \infty(\rightarrow$ stands for weak convergence).

Step two. We show that $x_{n} \rightarrow x^{*} \in \Omega$.

Since $x_{n+1}=\Pi_{K_{n+1}}^{p} x_{1} \subset K_{n+1} \subset K_{n}$ and $J^{p}$ is weakly sequentially continuous, we have by Lemma 1.11

$$
\begin{aligned}
\triangle_{p}\left(u_{n}, x_{n}\right) & =\triangle_{p}\left(u_{n}, x_{n+1}\right)+\triangle_{p}\left(x_{n+1}, x_{n}\right)+\left\langle u_{n}-x_{n+1}, J_{E_{1}}^{p} x_{n+1}-J_{E_{1}}^{p} x_{n}\right\rangle \\
& \leq \triangle_{p}\left(x_{n}, x_{n+1}\right)+\triangle_{p}\left(x_{n+1}, x_{n}\right)+\left\langle u_{n}-x_{n+1}, J_{E_{1}}^{p} x_{n+1}-J_{E_{1}}^{p} x_{n}\right\rangle \\
& =\triangle_{p}\left(x_{n}, x_{n}\right)+\left\langle u_{n}-x_{n}, J_{E_{1}}^{p} x_{n+1}-J_{E_{1}}^{p} x_{n}\right\rangle \\
& \longrightarrow 0 \text { as } n \rightarrow \infty .
\end{aligned}
$$

It follows from (2.1) that

$$
\frac{\left(J_{E_{1}}^{p} x_{n}-J_{E_{1}}^{p} u_{n}\right)-\lambda_{n} A^{*} J_{E_{2}}^{p}\left(I-\Pi_{A K}^{p} B_{\delta_{n}}^{T}\right) A x_{n}}{\delta_{n}} \in U\left(u_{n}\right) .
$$

By (2.17), we have that

$$
\triangle_{p}\left(u_{n}, v\right) \leq \triangle_{p}\left(x_{n}, v\right)-[1-\iota] \frac{\left\langle J_{E_{2}}^{p}\left(I-\Pi_{A K}^{p} B_{\delta_{n}}^{T}\right) A x_{n},\left(I-\Pi_{A K}^{p} B_{\delta_{n}}^{T}\right) A x_{n}\right\rangle}{\|A\|\left\|J_{E_{2}}^{p}\left(I-\Pi_{A K}^{p} B_{\delta_{n}}^{T}\right) A x_{n}\right\|},
$$

and

$$
\left\|\left(I-\Pi_{A K}^{p} B_{\delta_{n}}^{T}\right) A x_{n}\right\| \leq\left[\frac{\triangle_{p}\left(x_{n}, v\right)-\triangle_{p}\left(u_{n}, v\right)}{\|A\|^{-1}[1-\iota]}\right] \longrightarrow 0 \text { as } n \rightarrow \infty .
$$

By (2.23), we have that

$$
\triangle_{p}\left(u_{n}, v\right) \leq \triangle_{p}\left(x_{n}, v\right)-\frac{1}{p\|A\|^{p}} \frac{\left\langle J_{E_{2}}^{p}\left(I-\Pi_{A K}^{p} B_{\delta_{n}}^{T}\right) A x_{n},\left(I-\Pi_{A K}^{p} B_{\delta_{n}}^{T}\right) A x_{n}\right\rangle^{p}}{\left\|J_{E_{2}}^{p}\left(I-\Pi_{A K}^{p} B_{\delta_{n}}^{T}\right) A x_{n}\right\|^{p}}
$$

and therefore

$$
\left\|\left(I-\Pi_{A K}^{p} B_{\delta_{n}}^{T}\right) A x_{n}\right\| \leq\left[\frac{\triangle_{p}\left(x_{n}, v\right)-\triangle_{p}\left(u_{n}, v\right)}{(p\|A\|)^{-1}}\right]^{\frac{1}{p}} \longrightarrow 0 \text { as } n \rightarrow \infty .
$$

By (2.26) to (2.29) and weak sequential continuity property of $J^{p}$, we have that $0 \in U\left(x^{*}\right)$. This means that $x^{*} \in S O L V I P(U)$. But, since $\triangle_{p}(\cdot, x)$ is lower semi continuous and convex and thus weakly lower semi continuous on int(domf) then from the fact that $x_{n_{j}} \rightarrow x^{*}$ as $j \rightarrow \infty$, wee see that

$$
\triangle_{p}\left(x^{*}, x_{1}\right) \leq \liminf _{j \rightarrow \infty} \triangle_{p}\left(x_{n_{j}}, x_{1}\right) \leq \triangle_{p}\left(v, x_{1}\right)
$$

From the definition of $v$, that is $v=B_{\delta}^{U}(v)$, we can conclude that $x^{*}=v$ and the sequence $x_{n} \rightarrow x^{*}$. In addition, it is clear that $A x_{n} \rightarrow A x^{*}$. So by using (2.28), (2.29) and applying the demicloseness of $\left(I-\Pi_{A K}^{p} B_{\delta_{n}}^{T}\right)$ at zero, we have that $0 \in T\left(A x^{*}\right)$ as $\Pi_{A K}^{p} B_{\delta}^{T}\left(A x^{*}\right)=B_{\delta}^{T}\left(A x^{*}\right)$. Therefore $A x^{*} \in S O L V I P(T)$. Hence, $x^{*} \in \Omega$. 
Finally, by Lemma 1.11, we have

$$
\begin{aligned}
\limsup _{n \rightarrow \infty} \triangle_{p}\left(x_{n}, x^{*}\right) & =\limsup _{n \rightarrow \infty}\left[\triangle_{p}\left(x_{n}, x_{1}\right)+\triangle_{p}\left(x_{1}, x^{*}\right)+\left\langle x_{n}-x_{1}, J_{E_{1}}^{p} x_{1}-J_{E_{1}}^{p} x^{*}\right\rangle\right] \\
& \leq \limsup _{n \rightarrow \infty}\left[\triangle_{p}\left(x^{*}, x_{1}\right)+\triangle_{p}\left(x_{1}, x^{*}\right)+\left\langle x_{n}-x_{1}, J_{E_{1}}^{p} x_{1}-J_{E_{1}}^{p} x^{*}\right\rangle\right] \\
& =\limsup _{n \rightarrow \infty}\left\langle x^{*}-x_{n}, J_{E_{1}}^{p} x^{*}-J_{E_{1}}^{p} x_{1}\right\rangle=0 .
\end{aligned}
$$

Thus, we obtain $\lim _{n \rightarrow \infty} \triangle_{p}\left(x_{n}, x^{*}\right)=0$. Hence by Lemma 1.6 we get $x_{n} \rightarrow x^{*}$ as $n \rightarrow \infty$.

If $U: E_{1} \rightarrow E_{1}$ and $T: E_{2} \rightarrow E_{2}$ are nonexpansive in Theorem 2.3, then we get:

Corollary 2.4. For $\delta>0$ and $p, q \in(1, \infty)$, let $\left(I-\Pi_{A K}^{p} T\right)$ be demiclosed at zero. Let $x_{1} \in E_{1}$ be chosen arbitrarily and the sequence $\left\{x_{n}\right\}$ be defined as follows;

$$
\left\{\begin{array}{l}
u_{n}=U_{n}\left(J_{E_{1}^{*}}^{q}\left(J_{E_{1}}^{p} x_{n}-\lambda_{n} A^{*} J_{E_{2}}^{p}\left(I-\Pi_{A K}^{p} T_{n}\right) A x_{n}\right)\right), \\
K_{n+1}=\left\{v \in K_{n}: \triangle_{p}\left(u_{n}, v\right) \leq \triangle_{p}\left(x_{n}, v\right)\right\}, \\
x_{n+1}=\Pi_{K_{n+1}}^{p}\left(x_{1}\right), n \geq 1,
\end{array}\right.
$$

where

$$
\lambda_{n}= \begin{cases}\frac{1}{\|A\| \frac{1}{\left\|J_{E_{2}}^{p}\left(I-\Pi_{A K}^{p} T_{n}\right) A x_{n}\right\|},}, & x_{n} \neq 0 \\ \frac{1}{\|A\|^{p}} \frac{\left\langle J_{E_{2}}^{p}\left(I-\Pi_{A K}^{p} T_{n}\right) A x_{n},\left(I-\Pi_{A K}^{p} T_{n}\right) A x_{n}\right\rangle^{p-1}}{\left\|J_{E_{2}}^{p}\left(I-\Pi_{A K}^{p} T_{n}\right) A x_{n}\right\|^{p}}, & x_{n}=0,\end{cases}
$$

and $\mu_{n}=\frac{1}{\left\|x_{n}\right\|^{p-1}}$ are chosen such that

$$
\rho_{E_{1}^{*}}\left(\mu_{n}\right)= \begin{cases}\frac{\iota}{2^{q} G_{q}\|A\|} \times \frac{\left\langle J_{E_{2}}^{p}\left(I-\Pi_{A K}^{p} T_{n}\right) A x_{n},\left(I-\Pi_{A K}^{p} T_{n}\right) A x_{n}\right\rangle}{\left\|J_{E_{1}}^{p} x_{n}\right\|^{p}\left\|J_{E_{2}}^{p}\left(I-\Pi_{A K}^{p} T_{n}\right) A x_{n}\right\|}, & \text { if } \mu_{n} \in(0,1], \\ \frac{\iota}{2^{q} G_{q}\|A\|} \times \frac{\left\langle J_{E_{2}}^{p}\left(I-\Pi_{A K}^{p} T_{n}\right) A x_{n},\left(I-\Pi_{A K}^{p} T_{n}\right) A x_{n}\right\rangle}{\left\|J_{E_{2}}^{p}\left(I-\Pi_{A K}^{p} T_{n}\right) A x_{n}\right\|}, & \text { if } \mu_{n} \in(1, \infty),\end{cases}
$$

where $\iota \in(0,1)$. If $F(U)$ and $F\left(\Pi_{A K}^{p} T\right)$ denote the fixed point set of $U$ and $\Pi_{A K}^{p} T$, respectively, and $\Omega=\left\{x^{*} \in F(U) ; A x^{*} \in F\left(\Pi_{A K}^{p} T\right)\right\} \neq \emptyset$, then $\left\{x_{n}\right\}$ converges strongly to $x^{*} \in \Omega$, where $\Pi_{A K}^{p} T\left(A x^{*}\right)=T\left(A x^{*}\right)$.

Remark 2.5. Corollary 2.4 generalizes the corresponding results in $[6,7,11,15-17,22,23,28]$. In particular, it improves and extends the main result in [11] in the following aspects:

(1) we use a simpler algorithm,

(2) our split variational inclusion problem contains, as special case, their split feasibility problem,

(3) we work in a more general Banach space than $p$-uniformly convex.

In Theorem 2.3, let $\Pi_{A K}^{p}=\Pi_{A K}^{p} B_{\delta_{n}}^{T}$ and $\Pi_{K}^{p}=B_{\delta_{n}}^{U}$, where $\Pi_{K}^{p}: E_{1} \rightarrow K$ is the Bregman projection from $E_{1}$ onto $K$. Then we get the following result.

Corollary 2.6. For $\delta>0$ and $p, q \in(1, \infty)$, let $\Pi_{K}^{p}: E_{1} \rightarrow K$ be the Bregman projection from $E_{1}$ onto $K$ and $\left(I-\Pi_{A K}^{p}\right)$ be demiclosed at zero. Let $x_{1} \in E_{1}$ be chosen arbitrarily and the sequence $\left\{x_{n}\right\}$ be defined as follows;

$$
\left\{\begin{array}{l}
u_{n}=J_{E_{1}^{*}}^{q}\left(J_{E_{1}}^{p} x_{n}-\lambda_{n} A^{*} J_{E_{2}}^{p}\left(I-\Pi_{A K}^{p}\right) A x_{n}\right), \\
K_{n+1}=\left\{v \in K_{n}: \triangle_{p}\left(u_{n}, v\right) \leq \triangle_{p}\left(x_{n}, v\right)\right\}, \\
x_{n+1}=\Pi_{K_{n+1}}^{p}\left(x_{1}\right), n \geq 1,
\end{array}\right.
$$


where

$$
\lambda_{n}= \begin{cases}\frac{1}{\|A\| \frac{1}{\left\|J_{E_{2}}^{p}\left(I-\Pi_{A K}^{p}\right) A x_{n}\right\|},}, & x_{n} \neq 0 \\ \frac{1}{\|A\|^{p}} \frac{\left\langle J_{E_{2}}^{p}\left(I-\Pi_{A K}^{p}\right) A x_{n},\left(I-\Pi_{A K}^{p}\right) A x_{n}\right\rangle^{p-1}}{\left\|J_{E_{2}}^{p}\left(I-\Pi_{A K}^{p}\right) A x_{n}\right\|^{p}}, & x_{n}=0,\end{cases}
$$

and $\mu_{n}=\frac{1}{\left\|x_{n}\right\|^{p-1}}$ are chosen such that

$$
\rho_{E_{1}^{*}}\left(\mu_{n}\right)= \begin{cases}\frac{\iota}{2^{q} G_{q}\|A\|} \times \frac{\left\langle J_{E_{2}}^{p}\left(I-\Pi_{A K}^{p}\right) A x_{n},\left(I-\Pi_{A K}^{p}\right) A x_{n}\right\rangle}{\left\|J_{E_{1}}^{p} x_{n}\right\|^{p}\left\|J_{E_{2}}^{p}\left(I-\Pi_{A K}^{p}\right) A x_{n}\right\|}, & \text { if } \mu_{n} \in(0,1], \\ \frac{\iota}{2^{q} G_{q}\|A\|} \times \frac{\left\langle J_{E_{2}}^{p}\left(I-\Pi_{A K}^{p}\right) A x_{n},\left(I-\Pi_{A K}^{p}\right) A x_{n}\right\rangle}{\left\|J_{E_{1}}^{p} x_{n}\right\|^{p}\left\|J_{E_{2}}^{p}\left(I-\Pi_{A K}^{p}\right) A x_{n}\right\|}, & \text { if } \mu_{n} \in(0, \infty),\end{cases}
$$

where $\iota \in(0,1)$. If $\Omega=\left\{x^{*} \in K ; A x^{*} \in A K\right\} \neq \emptyset$, then $\left\{x_{n}\right\}$ converges strongly to $x^{*} \in \Omega$, where $\Pi_{A K}^{p} T\left(A x^{*}\right)=$ $T\left(A x^{*}\right)$.

Remark 2.7. Corollary 2.6 generalizes split feasibility problem result of Chen et al. [11] in the sense of Remark 2.5 (1) and (3). Moreover, this result, holds in a broader framework than a Hilbert space, so it generalizes the main result in [13].

Let $E=E_{1}=E_{2}$ be a Hilbert space, $I=J_{E_{1}}^{p}=J_{E_{2}}^{p}=J_{E_{1}^{*}}^{q}=A^{*}, p=q=2$, and let $U, T: E \rightarrow E$ be nonexpansive mappings. Suppose $F(U) \neq \emptyset$ and $F(T) \neq \emptyset$. The so-called hierarchical variational inequality problem for nonexpansive mapping $U$ with respect to a nonexpansive mapping $T$ is to find a point $x^{*} \in F(U)$ such that

$$
\left\langle x^{*}-T x^{*}, x^{*}-x\right\rangle \leq 0, \forall x \in F(U) .
$$

It is easy to see that (2.30) is equivalent to the following fixed point problem: find $x^{*} \in F(U)$ such that $A x^{*} \in F\left(P_{F(T)} T\right)$, where $P_{F(T)}: E \rightarrow F(T)$ is the metric projection from $E$ onto $F(T)$. Hence by Theorem 2.3, we deduce the following:

Corollary 2.8. For $\delta>0$, let $\left(I-P_{F(T)} T\right)$ be demiclosed at zero. Let $x_{1} \in E$ be chosen arbitrarily and the sequence $\left\{x_{n}\right\}$ be defined as follows;

$$
\left\{\begin{array}{l}
u_{n}=U_{n}\left(x_{n}-\lambda_{n}\left(I-P_{F(T)} T_{n}\right) x_{n}\right) \\
K_{n+1}=\left\{v \in K_{n}:\left\|u_{n}, v\right\| \leq\left\|x_{n}, v\right\|\right\} \\
x_{n+1}=P_{K_{n+1}}\left(x_{1}\right), n \geq 1
\end{array}\right.
$$

where

$$
\lambda_{n}= \begin{cases}\frac{1}{\left\|\left(I-P_{F(T)} T_{n}\right) x_{n}\right\|}, & x_{n} \neq 0 \\ 1, & x_{n}=0\end{cases}
$$

and $\mu_{n}=\frac{1}{\left\|x_{n}\right\|}$ are chosen such that

$$
\rho_{E}\left(\mu_{n}\right)= \begin{cases}\frac{\iota\left\|\left(I-P_{F(T)} T_{n}\right) x_{n}\right\|}{4 G_{2}\left\|x_{n}\right\|^{2}}, & \text { if } \mu_{n} \in(0,1], \\ \frac{\iota\left\|\left(I-P_{F(T)} T_{n}\right) x_{n}\right\|}{4 G_{2}}, & \text { if } \mu_{n} \in(0, \infty),\end{cases}
$$

where $\iota \in(0,1)$. If $F(U) \neq \emptyset$ and $F(T) \neq \emptyset$, then $\left\{x_{n}\right\}$ converges strongly to a solution of the hierarchical variational inequality problem (2.30), where $P_{F(T)} T\left(x^{*}\right)=T\left(x^{*}\right)$. 


\section{Application to split minimization problem}

The split minimization problem is to find:

$$
x^{*} \in E_{1} \text { such that } h\left(x^{*}\right) \leq h(x) \forall x \in E_{1}
$$

and

$$
A x^{*} \in E_{2} \text { such that } h^{\prime}\left(A x^{*}\right) \leq h^{\prime}(A x) \forall A x \in E_{2}
$$

where $h: E_{1} \rightarrow R$ and $h^{\prime}: E_{2} \rightarrow R$ are convex lower semicontinuous functions. Now let the subdifferential of $h$ and $h^{\prime}, \partial h: E_{1} \rightarrow 2^{E_{1}^{*}}$ and $\partial h^{\prime}: E_{2} \rightarrow 2^{E_{2}^{*}}$ be defined by

$$
(\partial h) x=\left\{x^{*} \in E_{1}^{*}: h(y)-h(x) \geq\left\langle y-x, x^{*}\right\rangle \forall y \in E_{1}\right\}
$$

and

$$
\left(\partial h^{\prime}\right) A x=\left\{A x^{*} \in E_{2}^{*}: h^{\prime}(A y)-h^{\prime}(A x) \geq\left\langle A y-A x, A x^{*}\right\rangle \forall A y \in E_{2}\right\},
$$

respectively.

It is well known that $\partial h$ and $\partial h^{\prime}$ are maximal monotone on $E_{1}$ and $E_{2}$ and that $0 \in(\partial h) x$ and $0 \in\left(\partial h^{\prime}\right) A x$ if $x$ and $A x$ are minimizers of $h$ and $h^{\prime}$, respectively. Hence

$$
B_{\delta}^{\partial h}=\operatorname{prox}_{\delta h} \text { and } B_{\delta}^{\partial h^{\prime}}=\operatorname{prox}_{\delta h^{\prime}} .
$$

In Theorem 2.3, $U=\partial h$ and $T=\partial h^{\prime}$, give the following result.

Theorem 3.1. Let the mapping of $\partial h, \partial h^{\prime}, \Pi_{A K}^{p}$, $\operatorname{prox}_{\delta h}$ and $\operatorname{prox}_{\delta h^{\prime}}$ be defined as above. For $\delta>0$ and $p$, $q \in(1, \infty)$, let $\left(I-\Pi_{A K}^{p} \operatorname{prox}_{\delta h^{\prime}}\right)$ be demiclosed at zero. Let $x_{1} \in E_{1}$ be chosen arbitrarily and the sequence $\left\{x_{n}\right\}$ be defined as follows;

$$
\left\{\begin{array}{l}
u_{n}=\operatorname{prox}_{\delta h}\left(J_{E_{1}^{*}}^{q}\left(J_{E_{1}}^{p} x_{n}-\lambda_{n} A^{*} J_{E_{2}}^{p}\left(I-\Pi_{A K}^{p} \operatorname{prox}_{\delta h^{\prime}}\right) A x_{n}\right)\right) \\
K_{n+1}=\left\{v \in K_{n}: \triangle_{p}\left(u_{n}, v\right) \leq \triangle_{p}\left(x_{n}, v\right)\right\} \\
x_{n+1}=\Pi_{K_{n+1}}^{p}\left(x_{1}\right), n \geq 1
\end{array}\right.
$$

where

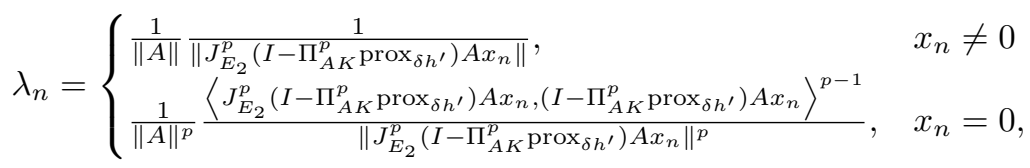

and $\mu_{n}=\frac{1}{\left\|x_{n}\right\|^{p-1}}$ are chosen such that

$$
\rho_{E_{1}^{*}}\left(\mu_{n}\right)= \begin{cases}\frac{\iota}{2^{q} G_{q}\|A\|} \times \frac{\left\langle J_{E_{2}}^{p}\left(I-\Pi_{A K}^{p} \operatorname{prox}_{\delta h^{\prime}}\right) A x_{n},\left(I-\Pi_{A K}^{p} \operatorname{prox}_{\delta h^{\prime}}\right) A x_{n}\right\rangle}{\left\|J_{E_{1}}^{p} x_{n}\right\|^{p}\left\|J_{E_{2}}^{p}\left(I-\Pi_{A K}^{p} \operatorname{prox}_{\delta h^{\prime}}\right) A x_{n}\right\|}, & \text { if } \mu_{n} \in(0,1], \\ \frac{\iota}{2^{q} G_{q}\|A\|} \times \frac{\left\langle J_{E_{2}}^{p}\left(I-\Pi_{A K}^{p} \operatorname{prox}_{\delta h^{\prime}}\right) A x_{n},\left(I-\Pi_{A K}^{p} \operatorname{prox}_{\delta h^{\prime}}\right) A x_{n}\right\rangle}{\left\|J_{E_{2}}^{p}\left(I-\Pi_{A K}^{p} \operatorname{prox}_{\delta h^{\prime}}\right) A x_{n}\right\|}, & \text { if } \mu_{n} \in(0, \infty),\end{cases}
$$

where $\iota \in(0,1)$. If $\Omega=\left\{x^{*} \in E_{1}: h\left(x^{*}\right) \leq h(x)\right.$ and $\left.h^{\prime}\left(A x^{*}\right) \leq h^{\prime}(A x), \forall x \in E_{1}\right\} \neq \emptyset$, then $\left\{x_{n}\right\}$ converges strongly to $x^{*} \in \Omega$, where $\Pi_{A K}^{p} \operatorname{prox}_{\delta h^{\prime}}\left(A x^{*}\right)=\operatorname{prox}_{\delta h^{\prime}}\left(A x^{*}\right)$. 


\section{A NUMERICAL EXAMPLE}

Let $E_{1}=E_{2}=\mathbb{R}, K=A K=[0, \infty)$ and $A x=x \forall x \in E_{1}$. Define

$$
\begin{aligned}
& U, T: \mathbb{R} \longrightarrow \mathbb{R} \text { by } U(x)=T(A x)=\left\{\begin{array}{l}
{[0,1], \quad x \geq 0} \\
\{1\}, \quad x<0,
\end{array}\right. \\
& P_{[0, \infty)}: \mathbb{R} \longrightarrow[0, \infty) \text { by } P_{[0, \infty)}(A x)= \begin{cases}0, & A x \in(-\infty, 0) \\
A x, & A x \in[0, \infty),\end{cases} \\
& (I+\delta U)^{-1}=(I+\delta T)^{-1}: \mathbb{R} \longrightarrow \mathbb{R} \\
& b y(I+\delta T)^{-1}(A y)=(I+\delta U)^{-1}(y)= \begin{cases}\frac{y}{1+[0, \delta]}, & y \geq 0 \\
\frac{y}{1+\delta}, & y<0,\end{cases} \\
& P_{[0, \infty)}(I+\delta T)^{-1}: \mathbb{R} \longrightarrow[0, \infty) \text { by } P_{[0, \infty)}(I+\delta T]^{-1}(A y)= \begin{cases}\frac{A y}{1+[0, \delta]}, & A y \geq 0 \\
0, & A y<0 .\end{cases}
\end{aligned}
$$

It is clear that $U$ and $T$ are multi-valued maximal monotone mappings such that $0 \in S O L V I P(U)$ and $0 \in S O L V I P(T)$. For $\delta_{n}=2^{n}$,

$$
\lambda_{n}= \begin{cases}\frac{\left|1+\left[0,2^{n}\right]\right|}{\left|x_{n}\left(1+\left[0,2^{n}\right]\right)-x_{n}\right|}, & x_{n}>0 \\ 1, & x_{n}=0, \\ \frac{1}{\left|x_{n}\right|}, & x_{n}<0\end{cases}
$$

we get that

$$
\begin{aligned}
u_{n} & = \begin{cases}\frac{x_{n}}{1+\left[0,2^{n}\right]}\left(x_{n}-1\right), & x_{n}>0, \\
0, & x_{n}=0, \\
\frac{x_{n}}{2^{n}+1}\left(x_{n}+1\right), & x_{n}<0,\end{cases} \\
K_{n+1}=\left\{v \in K_{n}: v \leq \frac{x_{n}-u_{n}}{2}\right\}, & \\
x_{n+1}= & P_{K_{n+1}} x_{1}= \begin{cases}\frac{x_{n}-\frac{x_{n}}{1+\left[2^{n}\right]}\left(x_{n}-1\right)}{2}, & x_{n}>0, \\
0, & x_{n}=0, \\
\frac{x_{n}-\frac{x_{n}}{2^{n}+1}\left(x_{n}+1\right)}{2}, & x_{n}<0 .\end{cases}
\end{aligned}
$$

In particular,

$$
x_{n+1}= \begin{cases}\frac{x_{n}-\frac{x_{n}}{\left(2^{n}+1\right)}\left(x_{n}-1\right)}{2}, & x_{n}>0, \\ 0, x_{n}=0, & \\ \frac{x_{n}-\frac{x_{n}}{2^{n}+1}\left(x_{n}+1\right)}{2}, & x_{n}<0 .\end{cases}
$$

Now by Theorem 2.3, the sequence $\left\{x_{n}\right\}$ converges strongly to $0 \in \Omega$. The Figures 1 and 2 below obtained by $(M A T L A B)$ software indicate convergence of $\left\{x_{n}\right\}$ given by $(2.1)$ with $x_{1}=1.0$ and $x_{1}=-1.0$, respectively. 


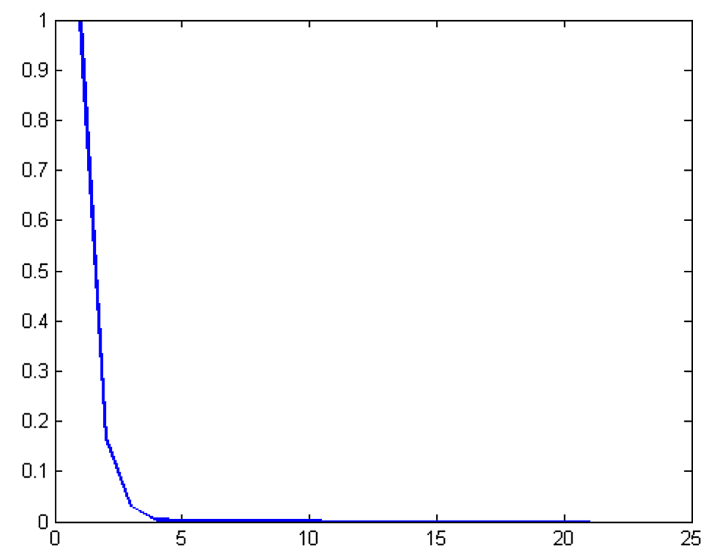

FIGURE 1. Convergence behavior of the sequence $\left\{x_{n}\right\}$ in $(2.1)$ with $x_{1}=1.0$.

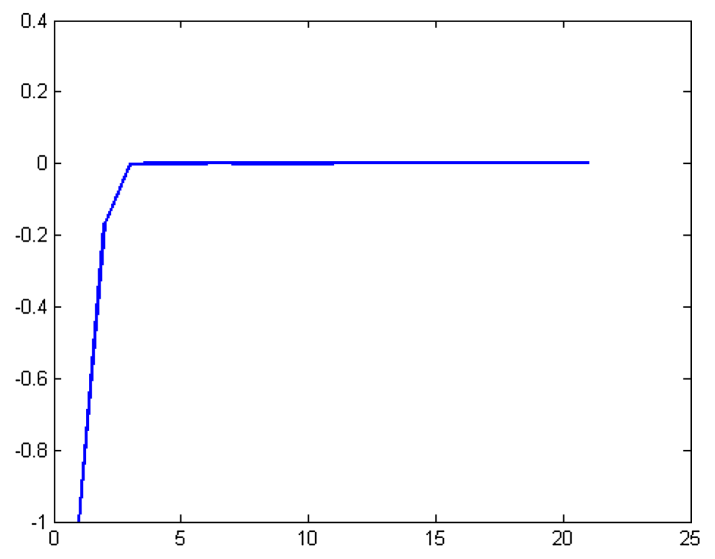

FiguRE 2. Convergence behavior of the sequence $\left\{x_{n}\right\}$ in $(2.1)$ with $x_{1}=-1.0$.

Acknowledgements. The author A.R. Khan would like to acknowledge the support provided by the Deanship of Scientific Research (DSR) at King Fahd University of Petroleum and Minerals (KFUPM) for funding this work through project No. IN141047.

\section{REFERENCES}

[1] C. Byrne, Iterative projection onto convex sets using multiple Bregman distances. Inverse Probl. 15 (1999) $1295-1313$.

[2] C. Byrne, A unified treatment of some iterative algorithms in signal processing and image reconstruction. Inverse Probl. $\mathbf{2 0}$ (2004) 103-120.

[3] C. Byrne, The split common null point problem. J. Nonlinear Convex Anal. 13 (2014) 759-775.

[4] V. Barbu, Maximal Monotone Operators in Banach Spaces. In: Nonlinear Differential Equations of Monotone Types in Banach Spaces. Springer Monographs in Mathematics. Springer, New York, NY (2011).

[5] A. Cegielski, Generalized relaxations of nonexpansive operators and convex feasibility problems. Contemp. Math. 513 (2010) $111-123$.

[6] L.C. Ceng, Q.H. Ansari and C.F. Wen, An extragradient method for solving split feasibility and fixed point problems. Comput. Math. Appl. 64 (2012) 633-642.

[7] L.C. Ceng, M.M. Wong, A. Petrusel and J.C. Yao, Relaxed implicit extragradient-like methods for finding minimum-norm solutions of the split feasibility problem. Fixed Point Theory 14 (2013) 327-344.

[8] Y. Censor and T. Elfving, A multiprojection algorithm using Bregman projections in product space. Numer. Algorithms 8 (1994) 221-239. 
[9] Y. Censor, T. Elfving, N. Kopf and T. Bortfeld, The multiple-sets split feasibility problem and its applications for inverse problems. Inverse Probl. 21 (2005) 2071-2084.

[10] Y. Censor, A. Gibali and S. Reich, Algorithms for the split variational inequality problem. Numer. Algorithms 59 (2012) 301-323.

[11] J.Z. Chen, H.Y. Hu and L.C. Ceng, Strong convergence of hybrid Bregman projection algorithm for split feasibility and fixed point problems in Banach spaces. J. Nonlinear Sci. Appl. 10 (2017) 192-204.

[12] M. Eslamian and P. Eslamian, Strong convergence of split common fixed point problem. Numer. Funct. Anal. Optim. 37 (2016) 1248-1266.

[13] A.R. Khan, M. Abbas and Y. Shehu, A general convergence theorem for multiple-set split feasibility problem in Hilbert spaces. Carpathian J. Math. 31 (2015) 349-357.

[14] B.M. Lawan and A. Kılıçman, Strong convergence for the split common fixed-point problem for total quasi-asymptotically nonexpansive mappings in Hilbert space. Abstr. Appl. Anal. 2015 (2015) 1-7.

[15] A. Moudafi, Split monotone variational inclusions. J. Optim. Theory Appl. 150 (2011) 275-283.

[16] A. Moudafi, The split common fixed-point problem for demicontractive mappings. Inverse Probl. 26 (2010) 1-6.

[17] K. Nakajo and W. Takahashi, Strong convergence theorem for nonexpansive mappings and nonexpansive semigroups. J. Math. Anal. Appl. 279 (2003) 372-379.

[18] S. Reich and S. Sabach, Two strong convergence theorems for Bregman strongly nonexpansive operators in reflexive Banach spaces. Nonlinear Anal. 73 (2010) 122-135.

[19] F. Schöpfer, Iterative regularization methods for the solution of the split feasibility problem in Banach spaces. Ph.D. Thesis, Universitat des Saarlandes (2007).

[20] F. Schöpfer, T. Schuster and A.K. Louis, An iterative regularization method for the solution of the split feasibility problem in Banach spaces. Inverse Probl. 24 (2008) 055008.

[21] W. Takahashi, The split common null point problem in Banach spaces. Arch. Math. (Basel) 104 (2015) $357-365$.

[22] W. Takahashi, Split feasibility problem in Banach spaces. J. Nonlinear Convex Anal. 15 (2014) 1349-1355.

[23] F.H. Wang, A new algorithm for solving multiple-sets split feasibility problem in Banach spaces. Numer. Funct. Anal. Optim. 35 (2014) 99-110.

[24] H.K. Xu, Existence and convergence for fixed points of mappings of asymptotically nonexpansive type. Nonlinear Anal. 16 (1991) 1139-1146.

[25] H.K. Xu, A variable Krasnoselskii-Mann algorithm and the multiple set split feasibility problem. Inverse Probl. 22 (2006) 2021-2034.

[26] H.K. Xu, Iterative methods for the split feasibility problem in infinite dimensional Hilbert spaces. Inverse Probl. 26 (2010) 105018.

[27] Z.-B. Xu and G.F. Roach, Characteristic inequalities of uniformly convex and uniformly smooth banach spaces. J. Math. Anal. App. 157 (1991) 189-210.

[28] Y.H. Yao, G. Marino and L. Muglia, A Korpelevich's method convergent to the minimum-norm solutions of a variational inequality. Optimization 63 (2012) 559-562.

[29] X.F. Zhang, L. Wang, L.M. Zhao and L.J. Qin, The strong convergence theorems for split common fixed point problem of asymptotically nonexpansive mappings in Hilbert spaces. J.Ineq. Appl. 2015 (2015) 1-11.

[30] J. Zhao and Q. Yang, Several solution methods for the split feasibility problem. Inverse Probl. 21 (2005) 1791-1800. 